\title{
A Pilot Policy Proposal to Improve WASH in Nigeria's Primary Healthcare to meet SDG 6 by 2030
}

\author{
Ogubuike Emejuru, MD, FAAP
}

Obinna O. Oleribe, MB,BS, DrPH, FRCP

\begin{abstract}
Nigeria is a country with over 190 million people spread across 36 states including the federal capital territory of the country. Although significant progress has been made towards strengthening the health system, the quality of Water Sanitation and Hygiene (WASH) services that results in Infection Prevention and Control (IPC) advancement efforts are lacking in most Primary Health Care (PHC) facilities in the country. For this reason, there are still gaps in attaining sustainable development goals (SDG -6). This pilot proposal if implemented will bring sustainable and improved WASH services that will improve IPC effort in Primary Health Care facilities. It will address bottleneck issues in leadership and governance and the health workforce which are major health system building blocks. Our target population is about 20 million vulnerable people living in and accessing PHC facilities in South-South, Nigeria. The proposal seeks to bridge the identified system gaps by strengthening leadership, governance, and workforce. This will be achieved by appointing WASH and IPC focal persons from the facilities and communities to improve community participation, accountability, and responsiveness. The pilot program will be scalable to the rest of the country. Liberia and Ethiopia are used as benchmarks references.
\end{abstract}

Key Words: Health Systems; Infection Prevention Control; Water Sanitation and Hygiene

\section{Introduction}

Nigeria is a lower-middle-income country and the most populous nation in Africa with a population above 190 million (National Population Commission, 2021). It has 36 states and the Federal Capital Territory is known as Abuja (Otekunrin, et al., 2019; World Bank, 2021). Nigeria has rich human and material resources but previously lacked good leadership and governance and a poorly remunerated health workforce regarding water, sanitation, and hygiene (WASH). The lack of adequate WASH services in most primary health care centers is a major public health issue. Inadequate WASH services have contributed immensely to the spread of Hospital Associated Infections (HAIs) in the country (Yaya et al., 2018).

Improved and adequate WASH services are vital components of a healthy primary health care (PHC) center's environment. Inadequate provision of WASH in healthcare settings leads to HAIs which poses significant health risks to people in and around the health facilities (Allegranzi et al., 2011; World Bank, 2020). In Nigeria, quality WASH services in PHC centers are inadequate (Ayekale, 2017; UNICEF, 2020; WaterAid, 2020). This has led to a high prevalence of HAIs. Meanwhile, in Sub- Saharan region, the prevalence of HAI varies between 2.5\% and 14.8\% (Irek et al., 2019; Sepideh et al, 2011). The prevalence of HAI in most public health facilities in Nigeria is high. The prevalence of HAIs such as pneumonia was found to be $1.9 \%$ and it constituted $12.0 \%$ of all the HAIs while gastrointestinal infections prevalence was 10.0\%. In all, the prevalence of HAIs ranged from $2.0 \%$ to $20.0 \%$ in the selected health facilities in the country (Abubakar, 2020). This reported high prevalence of HAIs is caused by poor access to WASH services emanating previously from lack 
of good leadership and governance and inadequate workforce. Thus, these challenges have limited the advancement of infection prevention and control efforts.

Nigeria operates a three (3) tiered health system (HS) namely: tertiary, secondary, and primary healthcare. The tertiary healthcare system provides highly specialized health services at teaching and specialist hospitals, the secondary healthcare system was created to cater for referrals from the primary healthcare system while the PHC system is responsible for health promotion, disease prevention, and other services at local government and ward level. (Raheem et al. 2019). In addition, PHC makes up about $85 \%$ of the nation's health facilities and Nigeria has about 30,000 PHC facilities situated in each ward. Most of these facilities are built in resource-constrained settings in the country where preventable WASHassociated infections are prevalent (Federal Ministry of Health (FMoH) 2016; Aigbiremolen et al. 2014; Ladi-Akinyemi et al. 2019).

Proposed Package Intervention

This proposed package program is a government-led intervention to strengthen WASH services in healthcare facilities in five (5) southern states in Nigeria. The focus is on improving WASH leadership and governance and health workforce in PHC facilities, further advancing IPC in Nigeria.

\section{Overarching Goal}

The overarching goal of this proposal is to develop a package of WASH interventions that would help to improve WASH leadership and governance, and the health workforce to advance IPC efforts in PHC facilities. The target population is about 20 million vulnerable people living in the South-South of Nigeria.

\section{Policy Objectives}

Set up a steering team

Strengthen WASH policy for improved WASH services in PHC

Embed and integrate WASH initiatives by building advocacy and coordination suitable for the reform program by 2026

Carryout bespoke capacity development and other ancillary training programs for 34 train the trainers focal persons on leadership and best hygiene practices

Ensure the workforce is mobilized and remunerated in each of the PHC facilities. 


\section{Review of Related Literature}

Two Health Systems Building Blocks

WASH is not just about infrastructure but entails good leadership and governance and a well remunerated health workforce. According to the United Nations (UN, 2020), having a functional leadership and governance and health workforce are integral parts of the global action plan or road map towards attaining universal access to WASH in health facilities and the sustainable development goal (SDG targets 6.1 and 6.2). Health leadership and governance are the administrative aspects of the health system that ensures strategic policy frameworks exist and are combined with effective oversight, coalition-building, regulation, attention to system design, and accountability.

Notably, the major target of leadership and governance is to achieve national policy objectives for effective delivery of services and the attainment of SDGs (Adeloye et al., 2017); while the health workforce is that component that consists of all personnel involved in activities solely devoted in improving health. Any nation without these components will not achieve any clinical and public health services. In Nigeria, poor leadership and governance and a poorly remunerated workforce have resulted in continued underinvestment in WASH services, which contributed immensely to weak systems that have led to an increased prevalence of HAIs in many PHC facilities (Oleribe et al., 2016; Yagboyaju, et al., 2019).

Bottleneck Issues

a) Leadership and Governance: Lack of political will and commitment by previous administrations, insufficient budgetary allocation due to lack of funds, insufficient infrastructure, poorly implemented policies that result in an increase in WASH-related diseases, lack of accountability, and lack of community/ stakeholders' engagement are major challenges in the country's Health System (HS).

b) Health workforce: Similarly, challenges in the health workforce contribute to weak HS. These challenges areinsufficient manpower required to maintain existing infrastructures, poorly incentivized workforce, poor attitude towards hygiene, and poorly trained workforce.

Nevertheless, while these were lacking in Nigeria previously, there are now concerted efforts towards strengthening and correcting these observed inadequacies. To strengthen WASH systems and reduce the risk of improper hygiene practices and hospital-acquired infections (HAIs), the World Health Organization and UNICEF, (2015) recommended standards. However, achieving these recommended standards (WHO, 2007) requires strong political will, adequate budgetary allocation, setting up institutional frameworks, and a strong health workforce. 
Addressing Issue by Reforms

To address these issues and show government commitment towards improving WASH services in institutions including health facilities, the government made some reforms. For example, the government declared a national emergency on water in 2018 and set up the Nigeria Sustainable Urban and Rural Water Supply, Sanitation, and Hygiene Program (SURWASH). This is being implemented as part of the National Action Plan (NAP) for the Revitalization of Nigeria's Water Supply, Sanitation, and Hygiene Sector and the National Urban Water Sector Reform Program (NUWSRP) (World Bank, 2020).

The National Action Plan (NAP) in accordance with SDG-6 is aimed at ensuring universal access to sustainable and safely managed WASH services by 2030. SUPERWASH was designed to supply 6 million people with basic drinking water services and 1.4 million people with access to improved sanitation services. Additionally, this program delivers improved WASH services to 2,000 schools and Health Care Facilities. At present, NAP and SURWASH are still at the implementation stages while NUWSRP has recorded good progress regarding the water sector reforms which has strengthened the capacity of the sector governance, institutions, and human capital development (World Bank, 2017). These successes were recorded because of government support and community participation and ownership.

Liberia

Insufficient WASH and IPC measures and personnel in health facilities during the Ebola outbreak accelerated the spread of Ebola. As a result, about 372 health workers acquired the virus during the initial outbreak, and 184 of these workers died. To strengthen the weak system in the area of workforce, the country introduced a focal WASH and infection control personnel.

Issues: Inadequate workforce

Solution: The Liberian health ministry engaged volunteers from the communities and used existing health personnel to bridge the manpower gap. The ministry trained and introduced WASH and IPC focal persons consisting of volunteers and staff into each of the 15 county's health management teams. The persons also served as environmental health officers working on WASH. These individuals oversaw the delivery of routine IPC measures and WASH interventions at healthcare facilities, in collaboration with the facility-based IPC officer. The individuals were also responsible for county outbreak preparedness and response efforts. This resulted in reduced cases and finally to the eradication of Ebola (Abrampah et al., 2017). 
Ethiopia

Ethiopia had leadership and governance challenges in its health system. This issue affected WASH services in most health facilities, leading to poor health outcomes. However, to improve WASH services and health outcomes, in 2014, the country started a Clean and Safe Health Facility (CASH) campaign.

Issue: Poor leadership and governance

Solution - Ethiopia's WASH focused on making hospitals safer by improving sanitation, hygiene, and patient safety. Notably, much of the focus was on facility-level activities (cleaning initiatives, staff and patient satisfaction surveys, and community engagement). High-level leadership such as government and community leaders were involved in the campaign. Their involvement led to the success of the program.

\section{Program Overview}

This program will be government-led action to improve WASH in healthcare facilities; it outlines the approaches that will be taken to strengthen health systems by focusing on good leadership and governance and a well remunerated workforce. The program aims to advance IPC efforts in PHC facilities in the South-South states of Nigeria. Furthermore, this intervention will ensure healthcare facilities in this region have safe, reliable, accessible, and inclusive WASH. Major reforms will be affected in these facilities using community volunteers as WASH ambassadors and ward managers as facility ambassadors. Hygiene and sanitation issues will be addressed by promoting behavioral changes through monthly training of community and facility WASH focal persons. Healthcare providers and other stakeholders will also be engaged.

The focal persons must commit to a three months intensive behavioral training program on sanitation and hygiene leadership. The program will adopt the social cognitive theory for the intervention because the theory is well known for designing health promotion interventions and is suitable for understanding health behaviors due to the interactions between individuals, environment, and behavior (Bandura, 2001). Further, the intervention strategies for the program will include five (5) phases, with each building upon the other to achieve the project goals. This will involve:

(1) Setting up a steering team to ensure the sustainability of the program

(2) Working with PHC board and other stakeholders to formulate and implement WASH and IPC policies.

(3) Advocacy to increase community ownership and participation in sanitation and hygiene practices in health facilities.

(4) Development of pre-service and in-service training materials regarding the importance of WASH to IPC advancement in health facilities and communities

(5) Engaging a well-trained workforce (ward managers and community ambassadors) in WASH and IPC facilitybased campaigns 
Program Description

This program seeks to improve sanitation and hygiene practices in 2000 PHC facilities in Southern Nigeria. This will be done through a combination of specialized training and general capacity building initiatives by identifying the needs of the workforce required and organizing quarterly training programs that will help to provide the skill set required. This program is scalable. It will be implemented in phases beginning from the Southern States. These states were chosen because of the insecurity and humanitarian crises that have plagued the region in recent times and have negatively affected PHC services, including WASH. People resident in these areas are in dire need of WASH services as most PHC either lacks WASH services or in centers where these services are available, the services are limited (UNICEF, 2018).

\section{Planned Activities}

1) Identification and selection of the steering committee: The members of the working committee will be identified and selected to carry out the designated functions.

2) Mobilization of Resources and Policy Formulation: Formulate final policies after consultation with sector stakeholders and then seek to obtain the necessary endorsements including legislative approvals. Two focal persons will be used as WASH and IPC Ambassadors champions. One will be the PHC manager (staff) while the other will be from the communities. The PHC managers will be rewarded using Performance-based incentives. The volunteer pool from the community may include community and religious leaders, administrative personnel, and media members. Leaders in the community will be used as ambassadors because of their influence in society. Both focal persons will bridge the health workforce gap and serve as the facility management team leaders.

3) Advocacy to increase community Ownership and Participation: There will be awareness campaigns every 4 months by way of advocacy to increase the community's participation and eventually take ownership of the processes, leading to accountability and a well-articulated and sustained sanitation and hygiene practice using the stated health facilities. This will help in proper coordination and increase access to quality and equitable WASH services. Additionally, it will strengthen the HS leadership and governance by increasing the knowledge on infection control and hygiene practices and help more citizens lend their voices on the need to provide and maintain quality WASH services

4) Capacity Building: Train the trainers (including the focal persons) will be identified and trained and properly equipped with the required skill set to train WASH and IPC personnel for effective service delivery, after which the trained personnel will conduct training across the 2000 PHC in Nigeria.

However, these local training will be continuously spread across pre-service and in-service training programs. The training and retraining will run all through the period of the program. A national curriculum on WASH will be introduced among tertiary institutions in collaboration with the ministry of education and these will be integrated into the program. It will further strengthen the health system workforce by improving behavioral changes around the facilities which in turn will result in improved WASH services and advancement of IPC. 
5) Review Meetings: Program team members and stakeholders will be expected to meet monthly and receive feedback from community and facility leaders. Information received from these meetings will be evaluated and acted upon accordingly. Numerous key stakeholders and focal persons will be identified as collaborators in the project. This is because much of the success and sustainability of the program goal depends on these collaborations.

\section{Program Output}

- $60 \%$ of the PHC centers have visionary leadership

- $\quad 80 \%$ of the PHC centers know WASH policies

- $80 \%$ of this policy is fully implemented in the PHC centers to achieve a safer and clean work environment

- $\quad 80 \%$ of the workforce are well trained on best practices on WASH and IPC in PHC and incentivized

- $\quad$ HAIs are reduced by $10 \%$ within twelve months of policy implementation.

Grounding in Behavioral Theory - Social Cognitive Theory

According to Bandura (2001), the social cognitive theory (SCT) is suitable for understanding health behaviors because of the interactions between individuals, environment, and behavior. Similarly, Glanz et al. (2014) assert that SCT summarizes different cognitive, emotional, and understandings of behavior change. The main constructs of SCT are selfefficacy and self-control. Outcome expectancy means beliefs related to a particular behavior that lead to specific results. After completing this training program designed using the principle underlying social cognitive theory, most participants are expected to change their behavior towards hygiene and sanitation in PHC centers.

\section{Sustainability}

This program will be sustained by improving the operational and financial efficiency of service provision, capacity building, ensuring community participation and ownership, ensuring that health managers that act as WASH focal persons are continuously incentivized through periodic monitoring and evaluation. Furthermore, the government plan to sustain the funds for this program by introducing and integrating a national health insurance scheme where companies and individuals will subscribe to insurance and pay a premium for health services, and the funds will be deployed to the health sector, including sustaining WASH and IPC services.

\section{Monitoring and Evaluation}

Monitoring and evaluation will be carried out using a continuous monitoring system. This will be implemented as part of project activities. Progress will be closely monitored through regular field visits to all PHC facilities and the participation of all key stakeholders involved to ensure timely and effective implementation. Monthly review meetings will be held highlighting the progress of the project, implementation, lessons learned, and the course corrections needed to 
address problems. The evaluation will assess the relevance, efficiency, effectiveness, outcome, and sustainability of program interventions. Monitoring and evaluation reports will be shared with USAID promptly.

\section{Policy Recommendation to meet SDG 6 by 2030 in Nigeria}

Access to safe drinking water and adequate sanitation is part of the SDGs-6. A review of development experience shows that the most effective way of achieving rapid and practical sustainable development is in improving the quality of life of the masses at the grassroots. Consequently, the focus of this proposal is improving WASH to advance IPC in the various PHC which make up about $85 \%$ of the nation's health facilities built-in resource-constrained settings; Best practice and the efficient use of resources to achieve SDG-6 require that Nigeria build a credible community and government-led systems that would improve WASH in PHC facilities. Research into best practice for WASH and IPC in PHC facilities have shown that well-resourced community-based and government-led services are the panaceas in a wide range of international settings. Therefore, concerted efforts are advised as follows:

A. Set up a dedicated SDG 6 working team at the Federal Ministry of Water Resources

B. Set up a scalable phased implementation to begin in a few specific communities in the country

C. Establish a steering team set up by policymakers to ensure the sustainability of the program.

D. The government should work with the PHC board and other stakeholders to formulate and implement WASH and IPC regulations

E. Appoint community WASH ambassadors to help strengthen the WASH workforce in PHC facilities

F. Incentives to be periodically provided to WASH staff based on their performance.

G. Government should prioritize WASH in the national budget.

H. Ensure that appropriate and relevant authorities promote transparency and accountability are instituted in every process

I. Support periodic advocacy to increase community ownership and participation in sanitation and hygiene practices and

J. Develop pre-service and in-service training materials to build capacity among the workforce.

\section{Conclusion}

Nigeria's 2015 MDG report acknowledges inadequate progress in the water and sanitation sector. There was some improvement in the provision of safe water but improvement in sanitation facilities remains a big challenge. The WASH Joint Monitoring Report puts this indicator at 29\%. (undp.org 2015). Nigeria is blessed with human resources and with political will, and early start, meeting the SDG- 6 by 2030 will be easy. 


\section{Ogubuike Emejuru, MD, FAAP}

Children's Hospital of the King's Daughters, Norfolk, Virginia

Eastern Virginia Medical School, Norfolk, Virginia

School of Public Health, University of Port Harcourt, Rivers State, Nigeria

Obinna O. Oleribe, DrPH, FRCP, MBA

School of Public Health, University of Port Harcourt, Rivers State, Nigeria

Public Health Consultant/Executive director

BEST HEALTH CONSULT (BHC) LLC

\section{References}

Abrampah, N. M., Montgomery, M., Baller, A., Ndivo, F., Gasasira, A., Cooper, C., \& Syed, S. B. (2017). Improving water, sanitation, and hygiene in health-care facilities, Liberia. Bulletin of the World Health Organization, 95(7), 526.

Abubakar, U. (2020). Point-prevalence survey of hospital-acquired infections in three acute care hospitals in Northern Nigeria. Antimicrobial Resistance \& Infection Control, 9, 1-7.

Adeloye, D., David, R. A., Olaogun, A. A., Auta, A., Adesokan, A., Gadanya, M., ... \& Iseolorunkanmi, A. (2017). Health workforce and governance: the crisis in Nigeria. Human resources for health, 15(1), 1-8.

Allegranzi.B, Bagheri Nejab.S, Combescure. C, Graafmans. W, Attar. H et al. (2011) Burden of endemic health-care-associated infection in developing countries: systematic review and meta-analysis. Lancet.(2011);377(9761):228-41.DOI:http://dx.doi.org/10.1016/S01406736(1 0)61458-4.

Federal Ministry of Health of Ethiopia (2005). National Infection Prevention Guidelines for Health care Facilities in Ethiopia. 1sted. Addis Ababa, Ethiopia: Disease Prevention and Control Department; (2005).

Federal Ministry of Health (2016). National health policy 2016. National Health Policy 2016 (naca.gov.ng)

Irek, E. O., Aliyu, A. A., Dahiru, T., Obadare, T. O., \& Aboderin, A. O. (2019). Healthcare-associated infections and compliance of hand hygiene among healthcare workers in a tertiary health facility, southwest Nigeria. Journal of infection prevention, 20(6), 289-296.

Ladi-Akinyemi, T. W., Daniel, O. J., Kanma-Okafor, O. J., Ogunyemi, A. O., \& Onajole, A. T. (2019). Assessment of health facilities, commodities, and supplies for malaria case management at primary healthcare centers in Ogun state, Nigeria. Journal of Medicine in the Tropics, $21(2), 56$.

National Population Commission (2021). Population statistics. National Population Commission - Providing Demographic Data For Social and Economic Planning For National Development.

Oleribe, O.O., Ezieme, I.P., Oladipo, O. et al. Industrial action by healthcare workers in Nigeria in 2013-2015: an inquiry into causes, consequences, and control—a cross-sectional descriptive study. Hum Resour Health 14, 46 (2016). https://doi.org/10.1186/s12960-016-0142-7

Oyekale, A. S. (2017). Assessment of primary health care facilities' service readiness in Nigeria. BMC health services research, 17(1), 1-12.

Pittet D, Allegranzi B, Storr J, Bagheri Nejad S, Dziekan G, Leotsakos A, et al. Infection control as a major World Health Organization priority for developing countries. J Hosp Infect 2008;68:285-92.

Raheem, T. Y., Adewale, B., Adeneye, A. K., Musa, A. Z., Ezeugwu, S. M. C., Yisau, J., ... \& Ujah, I. A. O. (2019). State of Health Facilities in Communities Designated for Community-Based Health Insurance Scheme in Nigeria: A Case Study of Kwara and Ogun States. International Journal of TROPICAL DISEASE \& Health, 1-10.

Samuel, S. O, Kayode, O. O, Musa, O. I, Nwigwe, G.C, Aboderin A.O, Salami T.A.T, et al. (2010) Nosocomial infections and the Challenges of control in developing countries. African Journal Of Clinical And Experimental Microbiology. (2010);11(2):102-10.

Stone, P. W. (2009). Economic burden of healthcare-associated infections: an American perspective. Expert review of pharmacoeconomics \& outcomes research, 9(5), 417-422.

Tietjen L., Bossemeyer D. and Baltimore, NM, (2003). Guidelines for Healthcare Facilities with Limited Resources: United States of America. JHPIEGO Corporation. Maryland: United State of America. 
United Nations Children Fund (2020), Water, Sanitation and Hygiene. Water, Sanitation, and Hygiene | UNICEF Nigeria

United Nations Children Fund (2018). Water, sanitation, and hygiene. Water, Sanitation, and Hygiene | UNICEF Nigeria

United Nations Children Fund (2020). WASH in health care facilities. WASH in health care facilities - UNICEF DATA

United Nations Children Fund (2018), WASH as a Cornerstone for Conquering the 2017 Cholera Outbreak in Borno State, Northeast Nigeria. Cholera Outbreak in Borno state.cdr (unicef.org)

United Nations (2020). Ensure availability and sustainability of the management of water and sanitation for all. Goal 6 | Department of Economic and Social Affairs (un.org)

UNDP (2015) undp.org/content/dam/undp/library/MDG/english/MDG\%20Country\%20Reports/Nigeria/Nigeria_MDGs_Abridged_Sept30.pdf

Water AID,( 2020), The scandal of $95 \%$ of healthcare centers in Nigeria and 150 million Nigerians without access to water and hygiene facilities in the face of the COVID-19 pandemic. The scandal of $95 \%$ of healthcare centers in Nigeria and 150 million Nigerians without access to water and hygiene facilities in the face of the COVID-19 pandemic | WaterAid Nigeria

World Bank (2017). Third National Urban Water Sector Reform Project (P123513). World Bank Document

World Bank (2021). Data from the lower-middle-income country, Nigeria. Data for Lower middle income, Nigeria | Data (worldbank.org)

World Bank (2021). Nigeria: ensuring water, sanitation, and hygiene for all. Nigeria: Ensuring Water, Sanitation and Hygiene for All (worldbank.org)

World Bank (2021). Improving water supply, sanitation, and hygiene services in Nigeria. Improving Water Supply, Sanitation and Hygiene Services in Nigeria (worldbank.org)

Yagboyaju, D. A. \& Akinola, A. O. (2019). Nigerian state and the crisis of governance: A critical exposition. Sage Open, 9(3), 2158244019865810.

Yaya, S., Hudani, A., Udenigwe, O., Shah, V., Ekholuenetale, M., \& Bishwajit, G. (2018). Improving water, sanitation and hygiene practices, and housing quality to prevent diarrhea among under-five children in Nigeria. Tropical medicine and infectious disease, $3(2), 41$. 